\title{
Vitamin D and Albuminuria in Youth with and without Type 1 Diabetes
}

\author{
Debika Nandi-Munshi $^{a} \quad$ Maryam Afkarian $^{b}$ Kathryn B. Whitlock ${ }^{c}$ \\ Jamie L. Crandell ${ }^{d}$ Ronny A. Bell ${ }^{\text {e Ralph D'Agostino }}{ }^{f}$ Sharon Saydah $^{g}$ \\ Amy K. Mottl ${ }^{\text {h }}$ Dana Dabelea ${ }^{i}$ Mary Helen Black ${ }^{j} \quad$ Elizabeth J. Mayer-Davisk \\ Catherine Pihoker' \\ ${ }^{a}$ Department of Pediatrics, University of Washington, Seattle, WA, USA; ${ }^{b}$ Nephrology Division, Department of \\ Medicine, University of California, Davis, CA, USA; ${ }^{\circ}$ Core for Biomedical Statistics, Center for Clinical \& Translational \\ Research, Seattle Children's Research Institute, Seattle, WA, USA; dDepartment of Biostatistics and School of \\ Nursing, University of North Carolina at Chapel Hill, Chapel Hill, NC, USA; ${ }^{\text {DDepartment }}$ of Public Health, \\ Brody School of Medicine, East Carolina University, Greenville, NC, USA; ${ }^{f}$ Department of Public Health Sciences, \\ Wake Forest School of Medicine, Winston-Salem, NC, USA; ${ }^{9}$ Division of Diabetes Translation, Centers for Disease

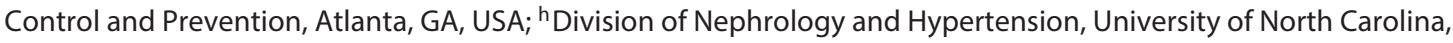 \\ Chapel Hill, NC, USA; 'Colorado School of Public Health, University of Colorado Denver, Aurora, CO, USA; \\ ${ }^{j}$ Ambry Genetics, Aliso Viejo, CA, USA; ${ }^{k}$ Departments of Nutrition and Medicine, University of North Carolina, \\ Chapel Hill, NC, USA; 'Department of Pediatrics, University of Washington, Seattle, WA, USA
}

\section{Keywords}

Vitamin D · Albuminuria · Type 1 diabetes · Children · Adolescents

\begin{abstract}
Background/Aims: In adults, lower vitamin D has been associated with increased albuminuria. This association has not been extensively studied in youth with or without type 1 diabetes. Methods: We examined the cross-sectional association between vitamin $\mathrm{D}$ and albuminuria (urine albumin to creatinine ratio $\geq 30 \mathrm{mg} / \mathrm{g}$ ) in 8,789 participants of the National Health and Nutrition Survey 2001-2006 (NHANES), who were 6-19 years old. Further, we examined the association between vitamin $\mathrm{D}$ and albuminuria in 938 participants from the SEARCH Nutritional Ancillary Study (SNAS), a longi-
\end{abstract}

tudinal cohort of youth with type 1 diabetes. Results: Of the NHANES participants, 5.3, 19.5, and 53.7\% had vitamin D levels <30, 50 and $80 \mathrm{nmol} / \mathrm{L}$, respectively. Albuminuria was present in $12.8 \%$ and was more common in younger children, females, non-Hispanic whites, non-obese children, and children with hypertension. After adjustments, there was no association between vitamin $D$ and albuminuria. Among the SNAS participants with type 1 diabetes, we also found no association between baseline vitamin $D$ and subsequent albuminuria in unadjusted or adjusted analyses. Conclusion: We did not find an association between serum vitamin $\mathrm{D}$ and albuminuria in either non-diabetic youth or those with type 1 diabetes. Further research is needed to more fully understand this relationship.

(c) 2017 S. Karger AG, Basel

\section{KARGER}

(c) 2017 S. Karger AG, Basel

E-Mail karger@karger.com

www.karger.com/hrp
Catherine Pihoker, MD

4800 Sandpoint Way NE, O.C. 7.820

Seattle, WA 98105 (USA)

E-Mail catherine.pihoker@ seattlechildrens.org 


\section{Introduction}

More than 190,000 people aged $<20$ years have diagnosed diabetes [1], and this number is projected to more than double by 2050 [2]. Among individuals with pediatric onset diabetes, $20-40 \%$ develop diabetic kidney disease (DKD) as adults [3-6]. Among people with diabetes, those with DKD have the highest risk of mortality [7, 8]. Increased albuminuria is the earliest available clinical indicator of $\mathrm{DKD}$, and a strong predictor of $\mathrm{DKD}$ progression, subsequent cardiovascular events, and mortality. In addition, in adults, a reduction of albuminuria by inhibition of the renin-angiotensin system (RAS) slows DKD progression, suggesting that albuminuria is not only an early indicator, but is also a potential therapeutic target in DKD.

Several lines of evidence connect low serum 25-hydroxyvitamin D (vitamin D) levels with increased risk of albuminuria. Animals deficient in vitamin $\mathrm{D}$ receptor develop more severe albuminuria and glomerulosclerosis [9], and this defect is reversed by expression of the vitamin D receptor in the kidney podocytes [10]. Vitamin D supplementation reduces glomerular hypertrophy, podocyte loss, glomerulosclerosis, and albuminuria in several models of kidney disease including DKD $[11,12]$. Furthermore, in animal models, vitamin D synergizes with RAS inhibitors to slow DKD progression [13-15].

In population-based studies, lower serum vitamin $\mathrm{D}$ levels have been linked to an increase in albuminuria in adults with or without diabetes [16-25]. In a large crosssectional study of adults without diabetes, an increase in the prevalence of albuminuria was observed with decreasing vitamin D concentration [17]. In a large prospective cohort study of individuals from the general population from Australia, the authors reported a higher incidence of albuminuria associated with vitamin $\mathrm{D}$ deficiency, and that this deficiency can independently predict future albuminuria [22]. However, an association of vitamin D and albumin excretion has not been consistently observed in cross-sectional [26] or longitudinal [27] studies. Activated vitamin $\mathrm{D}$ or its analogs have been shown to reduce albuminuria in adults with diabetes in several studies [28-32], including a randomized placebo-controlled trial [33]. A similar association between low vitamin D and albuminuria in children and adolescents has not yet been shown but would form the basis for evaluation of vitamin D supplementation as a potential novel therapy for DKD in children. Albuminuria is not uncommon in youth with diabetes; in the SEARCH for Diabetes in Youth study (SEARCH), albuminuria was present in $9.2 \%$ of youth with type 1 diabetes [34]. An advantage of long-term moderate vitamin D supplementation is that there are no known adverse effects.

The association between serum vitamin $\mathrm{D}$ and albuminuria in children and adolescents with diabetes has been examined in two previous studies. Lower vitamin D levels were associated with albuminuria in a small crosssectional study [35], but this was not replicated in a larger cross-sectional study [36]. In the current analysis, we evaluate the association between vitamin $\mathrm{D}$ and albuminuria in two large studies in children and adolescents with and without diabetes, using both cross-sectional and longitudinal analyses.

\section{Research Design and Methods}

\section{Study Populations}

The National Health and Nutrition Examination Survey (NHANES) is a nationally representative, population-based, crosssectional survey designed to provide estimates of health and nutritional status for the non-institutionalized population of the USA. NHANES 2001-2006 was approved by the Institutional Review Board at the Centers for Disease Control and Prevention's (CDC) National Center for Health Statistics. All participants underwent standardized in-home interviews, physical examinations, and laboratory testing at a mobile examination center. This study utilized data obtained in NHANES surveys conducted from 2001 to 2006. Serum vitamin $\mathrm{D}$ was measured in participants aged $\geq 6$ years in NHANES 2001-2002 and participants aged $\geq 1$ year in NHANES 2003-2006. Urine albumin to creatinine ratio (ACR) was measured in participants aged $\geq 6$ years in NHANES 2001-2006. Therefore, this study utilized data from NHANES 2001-2006 participants 6-19 years of age who had available serum vitamin D and urine albumin measurements $(n=8,789)$. Informed consent was obtained from all participants aged 18 years and older. Informed consent and parental permission was obtained from all participants aged 12-17 years and their parents. Assent and parental permission was obtained from all participants aged 7-11 years, and parental informed consent was obtained from all parents of participants aged $<7$ years. This study was not considered human subjects research by the University of Washington's Human Subjects Division because of the absence of identifying information and the publically available nature of the data.

The SEARCH Nutrition Ancillary Study (SNAS) is an ancillary study of the SEARCH study. The SEARCH study is a multicenter, observational, longitudinal study of youth with incident diabetes funded by the CDC with support from the National Institute of Diabetes and Digestive and Kidney Diseases (NIDDK). The study methods are described in detail in previous publications [37, 38]. Briefly, beginning in 2002 and continuing to the present, SEARCH has conducted population-based ascertainment of incident diabetes cases in youth aged $<20$ years at diagnosis at five locations (Ohio, Colorado, Washington, South Carolina, and Southern California). Youth with incident, non-secondary diabetes are invited to participate in an in-person visit, during which physical measurements, fasting blood, and urine samples are obtained. Youth 
with incident diabetes in the 2002-2005 SEARCH were invited to follow-up visits at 1, 2, and 5 years after their initial visit. For SEARCH participants with type 1 diabetes, defined by positive autoantibodies to glutamic acid decarboxylase 65 (GAD65) and/or insulinoma-associated peptide 2 (IA2) diagnosed in 2002-2005, SNAS collected additional nutritional data, including plasma measures of vitamin D obtained from frozen samples, which were collected at baseline. For this study, we utilized data from 938 SNAS participants with type 1 diabetes, whose diabetes duration was $\geq 3$ months at the baseline visit, who had completed a baseline visit in 2002-2005 and at least one follow-up visit subsequently, and had baseline vitamin $\mathrm{D}$, baseline and follow-up urine albumin, and creatinine measurements. Of these 938 participants, 66, 65.1, and $54.4 \%$ had a follow-up visit at 1,2 , and 5 years, respectively. The study was approved by the local institutional review board at each site. Written informed consent was obtained from all participants aged 18-19 years. Informed consent and parental assent were obtained from the parents or guardians of all participants $<18$ years of age and participants themselves, respectively, following particular study site Institutional Review Board regulations.

\section{Vitamin D Assessment}

Blood samples collected during the NHANES mobile examination center evaluations were centrifuged, aliquoted, and frozen at $-20^{\circ} \mathrm{C}$ on site. Samples were shipped on dry ice to the Division of Laboratory Sciences, National Center for Environmental Health at the CDC for analysis. Serum 25-hydroxyvitamin D was measured in thawed serum samples using a two-step extraction and radioimmunoassay using a kit from DiaSorin Inc. (Stillwater, MN, USA). Per NHANES analytical notes, vitamin D measurements for NHANES 2003-2004 and 2005-2006, but not NHANES 20012002, had to be adjusted for assay drift using a statistical adjustment model, based on data from a quality control pool which was collected during this interval [39].

In SNAS, blood samples were obtained after a 10 -h fast and under conditions of metabolic stability, defined as no episodes of diabetic ketoacidosis in the preceding month. The samples were centrifuged and shipped overnight on cold packs to the SEARCH central laboratory (University of Washington's Northwest Lipid Research Laboratory, Seattle, WA, USA). Upon receipt, the samples were aliquoted and stored at $-70^{\circ} \mathrm{C}$. Vitamin $\mathrm{D}$ was measured in thawed samples using the DiaSorin chemiluminescence immunoassay (Stillwater, MN, USA) at the Nutritional Biochemistry Core of the University of North Carolina's Nutrition and Obesity Research Center, based on a linkage between specific vitamin D antibody-coated magnetic particles and an isoluminol derivative. This method uses an antibody as a primary binding agent, measures $25(\mathrm{OH}) \mathrm{D} 2$ and $25(\mathrm{OH}) \mathrm{D} 3$, and has a detectable range of $5-320 \mathrm{nmol} / \mathrm{L}$ and an intra-assay coefficient of variation $(\mathrm{CV})$ of $11.0 \%$ [40]. There were 6 samples with undetectable levels of vitamin $\mathrm{D}$; these were imputed as $4.9 \mathrm{nmol} / \mathrm{L}$ because the lower limit of detection for the assay was $5 \mathrm{nmol} / \mathrm{L}$.

\section{Albuminuria Assessment}

In NHANES, spot urine samples collected during the mobile examination center evaluations were aliquoted and frozen at $-20^{\circ} \mathrm{C}$ on site. The samples were shipped on dry ice to the central laboratory at the University of Minnesota. Urine albumin and creatinine were measured in a single spot urine sample. Urine albumin was measured using a solid-phase fluorescent immunoassay on a fluorometer, with intra-assay and inter-assay CVs of $<8 \%$ [41]. Urine creatinine was measured using the kinetic Jaffe rate reaction [42] on a CX3 analyzer (Beckman ASTRA, Brea, CA, USA).

In SNAS, spot urine samples were obtained during the baseline and follow-up visits, after a 10 -h fast. The samples were not collected from participants who had a diabetic ketoacidosis in the preceding month, were pregnant or menstruating, had a fever or an acute infection, or had taken an antibiotic in the past week for a urinary tract infection. The samples were processed and shipped on cold packs overnight to the SEARCH central laboratory. Urine creatinine was measured in single spot urine samples using the Jaffe reaction on the Roche Modular P Autoanalyzer with an interassay $\mathrm{CV}$ of $<2 \%$. Urine albumin was measured using an immunochemical reaction on a Siemens BNII nephelometer, with an interassay CV of $5 \%$ and $7 \%$ for high- and low-level quality control samples, respectively.

In both of these studies, only spot urine samples were available. Urine albumin excretion in these samples was examined as ACR. Urine ACR was expressed in milligrams of albumin per grams of creatinine and was examined both as continuous and binary functional forms. When used as a continuous variable, it was log transformed to eliminate the marked right skew. As a binary variable, albuminuria was defined as a urine ACR $\geq 30 \mathrm{mg} / \mathrm{g}$.

For all analyses, individuals with nephrotic range proteinuria were excluded because of the known association between nephrotic proteinuria and vitamin D deficiency in children and adolescents [43-47].

\section{Covariates}

In NHANES, age, sex, and race/ethnicity were extracted from the participants' (or their parents' or guardians') responses to questionnaires, with race/ethnicity reported as non-Hispanic white, non-Hispanic black, Mexican-American, other Hispanic, or other [42]. Given the smaller sample sizes, Mexican-American and other Hispanic were combined for this study. Medications taken during the month prior to the NHANES physical examination were verified during in-person interviews and pill bottle review. Height and weight were measured during the physical examination and the body mass index (BMI) was calculated by dividing the weight (in $\mathrm{kg}$ ) by the height squared (in $\mathrm{m}^{2}$ ). The BMI z-score was calculated using the standardized CDC approach [48]. Overweight and obese were defined as a gender-and-age-specific BMI of 85th95th percentile and $>95$ th percentile, respectively, based on the year 2000 CDC US growth charts. Systolic and diastolic blood pressure (SBP and DBP) were ascertained from the average of $\geq 3$ resting measurements using a mercury sphygmomanometer by trained staff at the mobile examination center and during home examinations. Normal blood pressure was defined as SBP and DBP $<90$ th percentile. Pre-hypertension was defined as SBP or DBP $\geq 90$ th but $<95$ th percentile or a blood pressure $\geq 120 / 80$ but $<95$ th percentile. Hypertension was defined as SBP or DBP $\geq 95$ th percentile. Diabetes was defined as self-reported diagnosis of diabetes, hemoglobin A1c (A1c) $>6.5 \%$ (47.5 mmol $/ \mathrm{mol})$, or the use of antidiabetic medications. A1c measurements for participants in NHANES 2001-2004 were performed by the Diabetes Diagnostic Laboratory at the University of Missouri-Columbia using Primus CLC330 and Primus CLC 385 (Primus Corporation, Kansas City, MO, USA). Those for NHANES 2005-2006 were performed by the Diabetes Laboratory at the University of Minnesota using the 


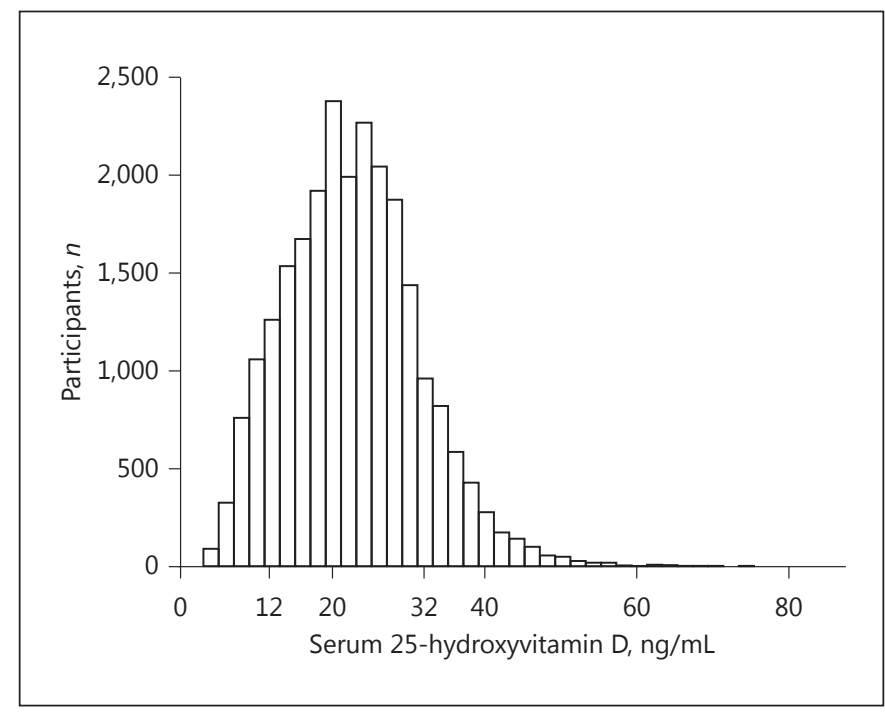

Fig. 1. Distribution of 25-hydroxyvitamin D in NHANES. Participants included children and adolescents 6-19 years of age, participating in NHANES 2001-2006, who had a serum vitamin D, urine albumin, and creatinine measurement available. Vitamin D levels of 12, 20, and $32 \mathrm{ng} / \mathrm{mL}$ correspond to thresholds of $<30,50$, and $80 \mathrm{nmol} / \mathrm{L}$ for vitamin $\mathrm{D}$ deficiency, respectively.

Tosoh A1c 2.2 Plus Glycohemoglobin Analyzer (Tosoh Medics, Inc., San Francisco, CA, USA). Both assays used a high-performance liquid chromatography platform and can be used without a cross-over regression, per NHANES analytical notes [49].

In SNAS, age, sex, and race/ethnicity (non-Hispanic white, non-Hispanic black, Hispanic, Asian/Pacific Islander, American Indian, and Multiple/other) were obtained from the responses of the participants or those of their parents or guardians to the SEARCH questionnaire. Height and weight were measured to the nearest $0.1 \mathrm{~cm}$ and $0.1 \mathrm{~kg}$, respectively. Age- and sex-specific BMI $\mathrm{Z}$-scores were used for statistical analyses [50]. Three blood pressure (BP) measurements were obtained using a portable mercury sphygmomanometer, and the average of these measurements yielded the BP measure assigned. Hypertension was defined as BP $>90 \%$ for age, sex, and height, or the use of antihypertensive medications. Type 1 diabetes diagnosis was confirmed by positivity for one or both of the auto-antibodies, GAD65 and IA2. These antibodies were measured using a radio-ligand binding assays, with cutoff values for positivity of $\geq 33$ and $\geq 5$ NIDDK U/mL for GAD65 and IA2, respectively [51]. A1c was measured using a dedicated ion-exchange high performance liquid chromatography instrument (Tosoh Bioscience).

\section{Statistical Analysis}

NHANES (cross-sectional) analyses were adjusted for survey design factors including cluster sampling (SDMVPSU), stratification (SDMVSTRA), and unequal probability weighting adjusted for the inclusion of multiple survey waves (WTMEC2YR/3). The subpopulation of interest, described above, was specified via domain and was not excluded outright from the analysis in order to preserve the accuracy of the published survey weights. Population estimates of mean vitamin $\mathrm{D}$ and prevalence of albuminuria were calculated overall and by age, sex, race/ethnicity, BMI category, diabetes status, and hypertensive status. The association of albuminuria with vitamin $\mathrm{D}$ was examined in logistic regression models without covariate adjustment (unadjusted); adjusted for age, sex, race/ethnicity, and BMI z-score (Model 1); and adjusted additionally for diabetes and hypertension status (Model 2). Sensitivity analyses were also conducted by assessing the association of vitamin $\mathrm{D}$ with continuous ACR, as well as after excluding participants with diabetes. Continuous ACR was log-transformed to improve distributional properties.

For the SNAS (longitudinal) analyses, baseline vitamin D and prevalence of albuminuria were summarized overall and by age, sex, race/ethnicity, BMI category, hypertension status, and A1c. The association of baseline vitamin $\mathrm{D}$ with subsequent albuminuria was examined using generalized estimating equations with a logit link and compound symmetry covariance structure to account for repeated measures per participant. Models included the covariates time since baseline (Base Model); additional covariates age, sex, race/ethnicity, and BMI categories (Model 1); and hypertension status and A1c (Model 2). Participants who met the criteria for albuminuria at baseline were excluded from the analysis. Sensitivity analyses were conducted in the same generalized estimating equation framework to further assess the association of vitamin D with (1) continuous ACR, log-transformed to improve distributional properties, logit link replaced with identity link; (2) albuminuria in a subset of participants with poor glycemic control, defined as $\mathrm{A} 1 \mathrm{c}>8 \%(63.9 \mathrm{mmol} / \mathrm{mol})$ at one or more time points; and (3) persistent albuminuria, defined as the occurrence of albuminuria in two or more follow-up visits in participants with no baseline albuminuria.

All analyses applied a significance level of $\alpha<0.05$ and were performed using SAS (version 9.12, SAS Institute, Cary, NC, USA).

\section{Results}

\section{Cross-Sectional Analyses Using NHANES}

Serum vitamin D was right-skewed with 5.3\% (95\% CI 4.0-6.6\%), 19.5\% (95\% CI 17.6-21.4\%), and 53.7\% (95\% CI 50.6-56.9\%) of all individuals having vitamin D levels $<12,20$, and $32 \mathrm{ng} / \mathrm{mL}$ (Fig. 1), respectively, corresponding to proposed thresholds of $<30,50$, and $80 \mathrm{nmol} / \mathrm{L}$ for vitamin D deficiency [52]. Statistically significant lower vitamin D levels were associated with older age, female sex, non-white race/ethnicity, obesity, diabetes, and hypertension. Overall, the prevalence of albuminuria was $12.8 \%$. Albuminuria was more common in younger children, females, non-Hispanic white, non-obese children, and children with hypertension (Table 1). The prevalence of albuminuria in children with diabetes (12.1\%) was similar to that in children without diabetes (12.8\%), likely because of the small number of youth with diabetes and low overall prevalence of albuminuria in this cohort. 
Table 1. Baseline characteristics of the NHANES 2001-2006 pediatric cohort and associations with 25-hydroxyvitamin D and albuminuria

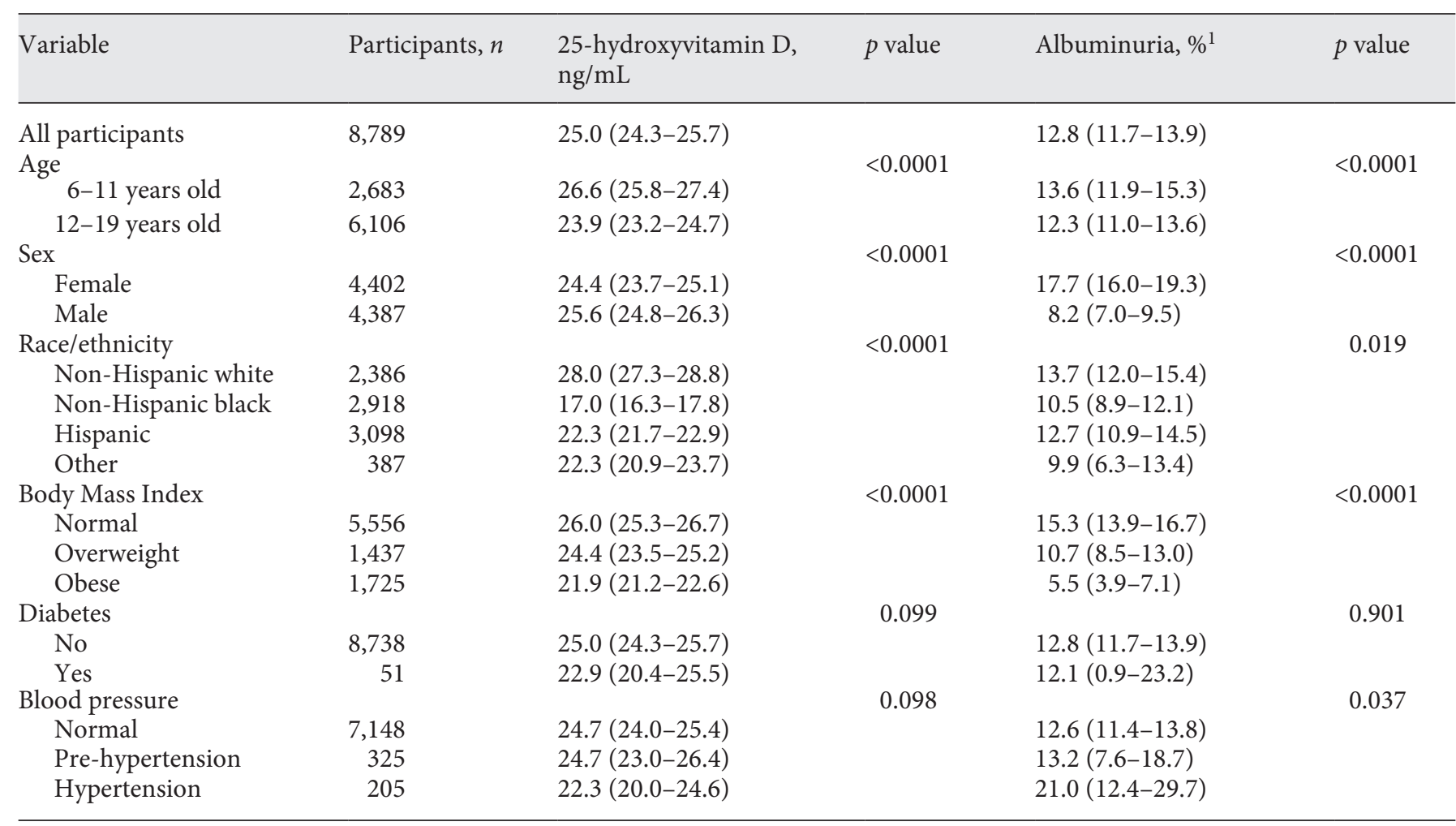

Values are expressed as means or prevalence with 95\% confidence interval in parentheses and are weighed to NHANES sampling distributions. ${ }^{1}$ Albuminuria is defined as an albumin to creatinine ratio $\geq 30 \mathrm{mg} / \mathrm{g}$. To convert 25 -hydroxyvitamin $\mathrm{D} \mathrm{in} \mathrm{ng} / \mathrm{mL}$ to $\mathrm{nmol} / \mathrm{L}$, multiply by 2.496. BMI categories: normal is $<85$ th percentile, overweight is $85-94$ th percentile, obese is $\geq 95$ th percentile. Blood pressure categories: normal is SBP and DBP $<90$ th percentile, pre-hypertension is SBP or DBP $\geq 90$ th percentile but $<95$ th percentile, hypertension is $\geq 95$ th percentile.

In unadjusted analysis, youth with higher serum vitamin D levels tended to have higher odds of albuminuria, though this association did not reach statistical significance (Fig. 2 and Table 2). After adjustment for demographic (age, sex, and race/ethnicity) and anthropometric (BMI z-score) variables, there was still no significant association between vitamin D and albuminuria (Table 2, Model 1). Further adjustment for blood pressure and diabetes (Table 2, Model 2), or exclusion of participants with diabetes ( $p$ value of 0.46 in Model 2) did not alter this finding. Evaluating ACR as a continuous variable did also not affect this finding (online suppl. Table 1; see www. karger.com/doi/10.1159/000475711 for all online suppl. material).

\section{Longitudinal Analysis Using SNAS}

In the SNAS cohort of children and adolescents with type 1 diabetes, serum vitamin $\mathrm{D}$ was right-skewed with $18.3,27.0$, and $31.8 \%$ of the participants having vitamin

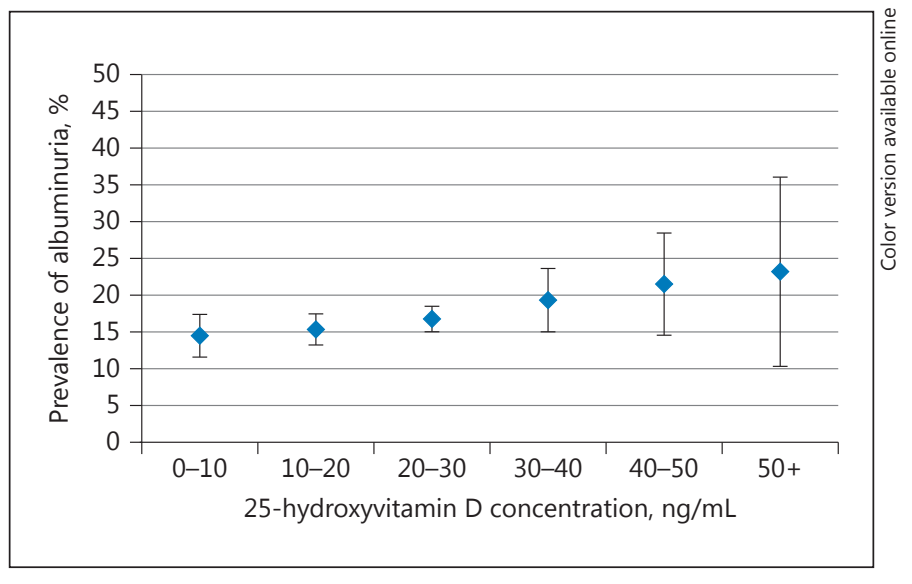

Fig. 2. Unadjusted prevalence of albuminuria by categories of circulating 25-hydroxyvitamin D in NHANES. 
Table 2. Association between 25-hydroxyvitamin $\mathrm{D}$ and albuminuria in NHANES 2001-2006

\begin{tabular}{llll}
\hline $\begin{array}{l}\text { Vitamin D, } \\
\text { ng/mL }\end{array}$ & Unadjusted & Model 1 & Model 2 \\
\hline $0-10$ & $0.79(0.49-1.25)$ & $1.20(0.72-2.01)$ & $1.27(0.75-2.14)$ \\
$10-20$ & $0.97(0.69-1.38)$ & $1.34(0.92-1.94)$ & $1.35(0.92-1.97)$ \\
$20-30$ & $1.07(0.78-1.46)$ & $1.22(0.91-1.65)$ & $1.18(0.85-1.63)$ \\
$30-40$ & $1.00($ Reference) & $1.00($ Ref. $)$ & $1.00($ Ref. $)$ \\
$40-50$ & $1.14(0.61-2.14)$ & $1.10(0.60-2.03)$ & $1.01(0.51-2.02)$ \\
$\geq 50$ & $1.62(0.64-4.08)$ & $1.57(0.64-3.83)$ & $1.80(0.74-4.35)$ \\
$p$ value & 0.37 & 0.56 & 0.38 \\
\hline
\end{tabular}

Albuminuria is defined as in Table 1. Effect estimates are expressed as odds ratio (95\% confidence interval). Model 1 was adjusted for age, sex, race/ethnicity, and BMI z-score. Model 2 was additionally adjusted for blood pressure status and diabetes, and thus limited to participants 8-19 years old for whom systolic and diastolic blood pressures were available. To convert 25-hydroxyvitamin $\mathrm{D}$ in $\mathrm{ng} / \mathrm{mL}$ to $\mathrm{nmol} / \mathrm{L}$, multiply by 2.496 . $p$ values assess overall association between vitamin $\mathrm{D}$ and albuminuria.

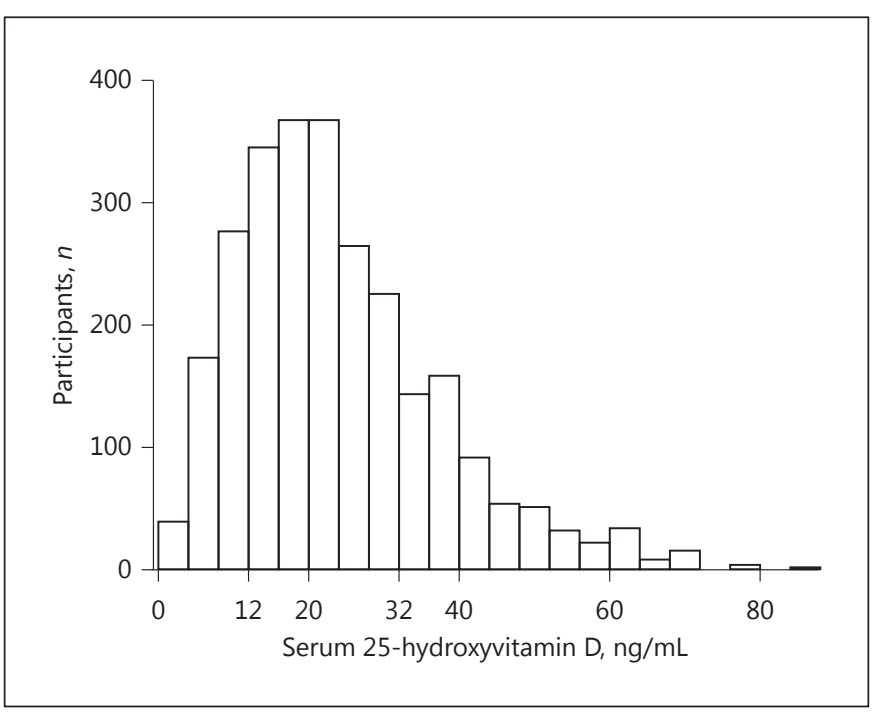

Fig. 3. Distribution of 25-hydroxyvitamin D in SNAS. The study population included SNAS participants with type 1 diabetes, diabetes duration of $>3$ months before the baseline visit, with baseline vitamin $\mathrm{D}$, baseline and follow-up urine albumin, and creatinine measurements. Vitamin D levels of 12,20 , and $32 \mathrm{ng} / \mathrm{mL}$ correspond to thresholds of $<30,50$, and $80 \mathrm{nmol} / \mathrm{L}$ for vitamin $\mathrm{D}$ deficiency, respectively.

D levels $<12,20$, and $32 \mathrm{ng} / \mathrm{mL}$ (Fig. 3 ), corresponding to thresholds of $<30,50$, and $80 \mathrm{nmol} / \mathrm{L}$ for vitamin $\mathrm{D}$ deficiency [52]. Similar to NHANES, lower vitamin D levels were associated with older age, non-white race/ethnicity, and obesity. Using the gender-blind ACR threshold, among the 938 SNAS participants studied here, 77 (8.2\%), 93 (9.9\%), and 170 (18.1\%) had albuminuria at the base- line, follow-up visit, or any visit, respectively. Albuminuria prevalence was higher in female children and those with poorly controlled diabetes (Table 3 ).

We found no significant association between baseline vitamin $\mathrm{D}$ and baseline albuminuria ( $p$ value of 0.62 in Model 2). There was also no association between baseline vitamin D and the subsequent development of albuminuria in unadjusted or adjusted analyses (Table 4). Participants with baseline albuminuria were excluded from this analysis; however, a sensitivity analysis including them did not alter these conclusions (data not shown). Evaluating ACR as a continuous variable did not affect this finding (online supp. Table 2). Furthermore, there was no significant association between vitamin $\mathrm{D}$ and subsequent albuminuria in the subgroup of SNAS participants with poor glycemic control in follow-up visits ( $p$ value of 0.13 in Model 2). In addition, we found no association between baseline vitamin $\mathrm{D}$ and subsequent persistent albuminuria ( $p$ value of 0.4 in Model 2).

\section{Discussion}

We found no association between serum vitamin D and albuminuria in children and adolescents, neither in a cross-sectional analysis of a nationally representative sample (NHANES 2001-2006) nor in a longitudinal analysis of a cohort of children and adolescents with type 1 diabetes (SNAS). This finding was consistently observed, whether the binary or continuous form of albuminuria was used. In the cohort with diabetes, this finding was also unaffected by the degree of glycemic control. 
Table 3. Baseline characteristics of the SNAS cohort and associations with 25-hydroxyvitamin D and albuminuria

\begin{tabular}{|c|c|c|c|c|c|}
\hline Variable & Participants, $n$ & $\begin{array}{l}\text { 25-hydroxyvitamin } \mathrm{D} \text {, } \\
\mathrm{ng} / \mathrm{mL}\end{array}$ & $p$ value & Albuminuria, $n(\%)$ & $p$ value \\
\hline All participants & 938 & $23.9(13.5)$ & & $77(8.2)$ & \\
\hline Age & & & 0.011 & & 0.1196 \\
\hline$<6$ years old & 102 & $26.2(14.1)$ & & $5(4.9)$ & \\
\hline 6-11 years old & 479 & $24.6(13.7)$ & & $35(7.3)$ & \\
\hline $12-19$ years old & 356 & $22.4(12.9)$ & & $37(10.4)$ & \\
\hline Female & 448 & $23.1(13.7)$ & & $48(10.7)$ & \\
\hline Male & 490 & $24.6(13.4)$ & & $29(5.9)$ & \\
\hline Race/ethnicity & & & $<0.0001$ & & 0.8322 \\
\hline Non-Hispanic white & 732 & $25.3(13.7)$ & & $58(7.9)$ & \\
\hline Non-Hispanic black & 85 & $15.5(9.3)$ & & $8(9.4)$ & \\
\hline Other & 121 & $21.4(12.7)$ & & $11(9.1)$ & \\
\hline Blood pressure & & & 0.8802 & & 0.4263 \\
\hline Normal & 928 & $24.0(13.6)$ & & $77(8.3)$ & \\
\hline Hypertension & 7 & $23.2(13.3)$ & & $0(0)$ & \\
\hline Hemoglobin A1c & & & 0.5306 & & 0.0169 \\
\hline$\leq 8 \%$ & 615 & $24.1(13.6)$ & & $41(6.7)$ & \\
\hline$>8 \%$ & 322 & $23.5(13.4)$ & & $36(11.2)$ & \\
\hline
\end{tabular}

Values are expressed either as means with SD in parentheses or prevalence in numbers with percent in parentheses. Albuminuria is defined as in Table 1 . To convert 25 -hydroxyvitamin $\mathrm{D}$ in $\mathrm{ng} / \mathrm{mL}$ to $\mathrm{nmol} / \mathrm{L}$, multiply by 2.496 . BMI categories: normal is <85th percentile, overweight is $85-94$ th percentile, obese is $\geq 95$ th percentile. Hypertension was defined as blood pressure $>90 \%$ for age, sex, and height, or the use of antihypertensive medications.

Table 4. Association between baseline 25-hydroxyvitamin D and subsequent albuminuria in the SNAS cohort

\begin{tabular}{lccc}
\hline $\begin{array}{l}\text { Vitamin D, } \\
\text { ng/mL }\end{array}$ & Unadjusted & Model 1 & Model 2 \\
\hline $0-20$ & $1.12(0.56-2.26)$ & $1.13(0.56-2.28)$ & $1.07(0.51-2.24)$ \\
$20-30$ & $1.26(0.59-2.70)$ & $1.22(0.57-2.62)$ & $1.24(0.56-2.73)$ \\
$30-40$ & $1.00($ Reference) & 1.00 (Reference) & 1.00 (Reference) \\
$40-50$ & $0.84(0.25-2.82)$ & $0.76(0.22-2.59)$ & $0.73(0.21-2.58)$ \\
$\geq 50$ & $1.57(0.55-4.51)$ & $1.39(0.47-4.13)$ & $1.59(0.51-5.02)$ \\
$p$ value & 0.87 & 0.88 & 0.80 \\
\hline
\end{tabular}

Albuminuria is defined as in Table 1. Effect estimates are expressed as odds ratio (95\% confidence interval). The groups with 25 -hydroxyvitamin $\mathrm{D}<20$ were combined due to the small sample numbers in each group. Model 1 was adjusted for age, sex, race/ethnicity, and BMI z-score. Model 2 was additionally adjusted for hypertension and hemoglobin A1c. To convert 25-hydroxyvitamin $\mathrm{D}$ in $\mathrm{ng} / \mathrm{mL}$ to $\mathrm{nmol} / \mathrm{L}$, multiply by 2.496 . $p$ values assess overall association between vitamin $\mathrm{D}$ and albuminuria.
Lower vitamin $\mathrm{D}$ levels have previously been shown to be strongly associated with albuminuria in adults [1625]. While children and adolescents with type 1 diabetes appear to have lower circulating vitamin D levels than the general population [53-55], our findings suggest that these lower vitamin D levels are not associated with higher rates of albuminuria. 
Examination of albuminuria in children and adolescents is fraught with several difficulties. The definition of albuminuria in pediatric cohorts continues to rely on a non-validated gender-blind definition, which overestimates albuminuria in girls because of their lower daily creatinine excretion [56-58]. To address this problem, we examined vitamin $\mathrm{D}$ association with the continuous form of ACR and observed similar results to those obtained from binary albuminuria. While the continuous ACR may still overestimate the absolute albumin excretion in girls, the lack of a binary threshold helps to avoid misclassification of a subset of girls with high normal albumin excretion as albuminuria. However, we found no association between vitamin $\mathrm{D}$ and the continuous ACR.

Several explanations are possible for why we found no association between circulating vitamin $\mathrm{D}$ and albuminuria in children. Firstly, albuminuria in children appears to be clinically dissimilar from that in adults. Unlike in adults, the vast majority (75-95\%) of albuminuria in children and adolescents is transient, occurring due to causes such as orthostatic proteinuria, which have not been associated with structural kidney disease and have benign outcomes in the general population $[59,60]$. This may explain the observation that not only micro- but also macroalbuminuria is highly reversible in children with diabetes, even without treatment with RAS inhibitors $[61,62]$. If only the pathogenic cases of albuminuria are associated with low circulating vitamin $\mathrm{D}$ and the majority of albuminuria observed in our study populations is benign, even a large sample size such as those utilized here may lack adequate power to detect this association. As an aside, it is worth noting that the contribution of orthostatic or transient albuminuria to kidney disease in children with diabetes is not known. Secondly, it is possible that even pathogenic albuminuria in children is not associated with low circulating vitamin D, suggesting that some of its underlying mechanisms may be different from those in adults. Distinguishing these possibilities would require examining urine albumin excretion in samples collected in the absence of conditions causing transient and orthostatic proteinuria, so that truly pathogenic proteinuria can be identified, without misclassification from benign causes. This highlights the difficulty of interpreting albuminuria in the majority of currently available pediatric cohorts and populations, where urine samples are collected under conditions that do not allow this distinction. It also emphasizes the importance of accounting for these factors in future pediatric studies. In light of these findings, since 2011 the
SEARCH cohort has collected urine samples under conditions which would exclude orthostatic and transient albuminuria (e.g. collection of first morning void, urine not collected after a fever, strenuous exercise, etc.). Future examination of samples and data collected under these conditions may shed additional light on mechanism and associations of albuminuria in children with diabetes.

This study is subject to several limitations. Firstly, urine albumin excretion was ascertained in random (not timed) urine samples and in samples that were collected under conditions which could allow misclassification by transient and orthostatic proteinuria (as discussed above). Secondly, serum vitamin D was quantified only at baseline. Additionally, the follow-up duration in SNAS was short. Finally, the analyses of persistent albuminuria excluded $11.5 \%$ of the SNAS participants who had baseline albuminuria or inadequate follow-up. Like other studies of albuminuria in children, this study is limited by a lack of existing age-, sex-, and race-specific reference values for albuminuria in children and adolescents. We also acknowledge limitations of cross-sectional studies for the evaluation of association between an exposure and a chronically occurring outcome, such as albuminuria. It is also important to note that studying individuals with a longer duration of diabetes than 5 years may have yielded different results, as the occurrence of microalbuminuria rises significantly after 10 or more years' duration.

Despite these limitations, this study is the first to evaluate the association between vitamin $\mathrm{D}$ and albuminuria cross-sectionally in a large general population of children within a nationally representative study as well as longitudinally in a multicenter cohort of children with type 1 diabetes, lending the data wide external generalizability. Additional strengths are the uniform assessment of albuminuria and vitamin D within each study (NHANES and SNAS), meticulous diabetes characterization in SNAS, and the examination of continuous as well as binary measurement of albuminuria. Furthermore, though these studies date back to the early 2000s, they were conducted under the same standards of care which are currently in use, which makes these findings relevant to current clinic practice.

In conclusion, we find no evidence of association between circulating 25-hydroxyvitamin D and albuminuria in cross-sectional or longitudinal analyses of large nationally representative populations of children and adolescents with or without type 1 diabetes. 


\section{Acknowledgements}

The SEARCH for Diabetes in Youth Study and the SEARCH Nutrition Ancillary Study is indebted to the many youth and their families, and their health care providers, whose participation made this study possible.

The authors wish to acknowledge the involvement of the South Carolina Clinical and Translational Research Institute, at the Medical University of South Carolina, NIH/National Center for Advancing Translational Sciences (NCATS) grant number UL1 TR001450; Seattle Children's Hospital and the University of Washington, NIH/NCATS grant number UL1 TR00423; University of Colorado Pediatric Clinical and Translational Research Center, NIH/NCATS grant number UL1 TR000154; the Barbara Davis Center at the University of Colorado at Denver (DERC NIH grant number P30 DK57516); the University of Cincinnati, NIH/ NCATS grant number UL1 TR001425; and the Children with Medical Handicaps program managed by the Ohio Department of Health. This study includes data provided by the Ohio Department of Health, which should not be considered an endorsement of this study or its conclusions.

The authors wish to acknowledge the work of the University of North Carolina Nutrition Obesity Research Center for conduct of the vitamin D assays (NIH DK056350) and the involvement of General Clinical Research Centers (GCRC) at the South Carolina Clinical and Translational Research (SCTR) Institute, at the Medical University of South Carolina (NIH/NCRR grant number UL1RR029882); Children's Hospital and Regional Medical Center (grant number M01RR00037); Colorado Pediatric General Clinical Research Center (grant number M01 RR00069) and the Barbara Davis Center at the University of Colorado at Denver (DERC NIH P30 DK57516); and the Institutional Clinical and Translational Science Award (CTSA), NIH/NCRR at the University of Cincinnati (grant number 1UL1RR026314-01).

The findings and conclusions in this report are those of the authors and do not necessarily represent the official position of the Centers for Disease Control and Prevention and the National Institute of Diabetes and Digestive and Kidney Diseases.

\section{Funding Sources}

This study was supported by a Pediatric Pilot and Fund from the Center for Clinical and Translational Research at Seattle Children's Hospital (to C.P.), career development K23DK089017 from the NIDDK (to M.A.), and Norman S. Coplon Extramural Grant from Satellite Healthcare (to M.A.).

The SEARCH for Diabetes in Youth Cohort Study (1UC4DK108173-01) is funded by the National Institutes of Health, National Institute of Diabetes and Digestive and Kidney Diseases and supported by the Centers for Disease Control and Prevention.

The Population Based Registry of Diabetes in Youth Study (RFP DP15-002) is funded by the Centers for Disease Control and Prevention and supported by the National Institutes of Health, National Institute of Diabetes and Digestive and Kidney Diseases. The SEARCH Nutrition Ancillary Study is funded by NIH/NIDDK R01 DK077949 (principal investigator: E.J.M.-D.).

Site Contract Numbers: Kaiser Permanente Southern California (U18DP006133, U48/CCU919219, U01 DP000246, and U18DP002714), University of Colorado Denver (U18DP006139, U48/CCU819241-3, U01 DP000247, and U18DP000247-06A1), Cincinnati's Children's Hospital Medical Center (U18DP006134, U48/CCU519239, U01 DP000248, and 1U18DP002709), University of North Carolina at Chapel Hill (U18DP006138, U48/ CCU419249, U01 DP000254, and U18DP002708), Seattle Children's Hospital (U18DP006136, U58/CCU019235-4, U01 DP000244, and U18DP002710-01), Wake Forest University School of Medicine (U18DP006131, U48/CCU919219, U01 DP000250, and 200-2010-35171).

\section{Author Contribution}

M.A. and C.P. conceived the study idea. M.A. and D.N.-M. researched the background material. M.A., D.N.-M. and C.P. designed the studies. K.B.W. analyzed the data. M.A., D.N.-M. and C.P. reviewed the data. M.A. wrote the manuscript. All authors actively participated in critical review of the manuscript and have read and approved the final version of the manuscript. C.P. and M.A. are the guarantors of this study, had full access to all the data, and take full responsibility for the integrity of the data and accuracy of the analyses.

\section{References}

1 Pettitt DJ, Talton J, Dabelea D, Divers J, Imperatore G, Lawrence JM, Liese AD, Linder B, Mayer-Davis EJ, Pihoker C, Saydah SH, Standiford DA, Hamman RF; SEARCH for Diabetes in Youth Study Group: Prevalence of diabetes in U.S. youth in 2009: the SEARCH for diabetes in youth study. Diabetes Care 2014; 37:402-408.

2 Imperatore G, Boyle JP, Thompson TJ, Case D, Dabelea D, Hamman RF, Lawrence JM, Liese AD, Liu LL, Mayer-Davis EJ, Rodriguez BL, Standiford D; SEARCH for Diabetes in Youth Study Group: Projections of type 1 and type 2 diabetes burden in the U.S. population aged $<20$ years through 2050: dynamic mod- eling of incidence, mortality, and population growth. Diabetes Care 2012;35:2515-2520.

$\checkmark 3$ Andersen AR, Christiansen JS, Andersen JK, Kreiner S, Deckert T: Diabetic nephropathy in type 1 (insulin-dependent) diabetes: an epidemiological study. Diabetologia 1983;25: 496-501.

4 Wong TY, Shankar A, Klein R, Klein BE: Retinal vessel diameters and the incidence of gross proteinuria and renal insufficiency in people with type 1 diabetes. Diabetes 2004;53: 179-184.

5 Molitch ME, DeFronzo RA, Franz MJ, Keane WF, Mogensen CE, Parving HH, Steffes MW, American Diabetes Association: Nephropa- thy in diabetes. Diabetes Care 2004;27(suppl 1):S79-S83

6 Hovind P, Tarnow L, Rossing P, Jensen BR, Graae M, Torp I, Binder C, Parving HH: Predictors for the development of microalbuminuria and macroalbuminuria in patients with type 1 diabetes: inception cohort study. BMJ 2004;328:1105.

7 Groop PH, Thomas MC, Moran JL, Waden J, Thorn LM, Makinen VP, Rosengard-Barlund M, Saraheimo M, Hietala K, Heikkila O, Forsblom C, FinnDiane Study Group: The presence and severity of chronic kidney disease predicts all-cause mortality in type 1 diabetes. Diabetes 2009;58:1651-1658. 
8 Orchard TJ, Secrest AM, Miller RG, Costacou $\mathrm{T}$ : In the absence of renal disease, 20 year mortality risk in type 1 diabetes is comparable to that of the general population: a report from the Pittsburgh Epidemiology of Diabetes Complications Study. Diabetologia 2010; 53:2312-2319.

-9 Zhang Z, Sun L, Wang Y, Ning G, Minto AW, Kong J, Quigg RJ, Li YC: Renoprotective role of the vitamin $\mathrm{D}$ receptor in diabetic nephropathy. Kidney Int 2008;73:163-171.

10 Wang Y, Deb DK, Zhang Z, Sun T, Liu W, Yoon D, Kong J, Chen Y, Chang A, Li YC: Vitamin $\mathrm{D}$ receptor signaling in podocytes protects against diabetic nephropathy. J Am Soc Nephrol 2012;23:1977-1986.

11 Schwarz U, Amann K, Orth SR, Simonaviciene A, Wessels S, Ritz E: Effect of 1,25 $(\mathrm{OH}) 2$ vitamin $\mathrm{D} 3$ on glomerulosclerosis in subtotally nephrectomized rats. Kidney Int 1998;53:1696-1705.

12 Kuhlmann A, Haas CS, Gross ML, Reulbach U, Holzinger M, Schwarz U, Ritz E, Amann K: 1,25-Dihydroxyvitamin D3 decreases podocyte loss and podocyte hypertrophy in the subtotally nephrectomized rat. Am J Physiol Renal Physiol 2004;286:F526-F533.

13 Zhang Y, Deb DK, Kong J, Ning G, Wang Y, Li G, Chen Y, Zhang Z, Strugnell S, Sabbagh Y, Arbeeny C, Li YC: Long-term therapeutic effect of vitamin $\mathrm{D}$ analog doxercalciferol on diabetic nephropathy: strong synergism with AT1 receptor antagonist. Am J Physiol Renal Physiol 2009;297:F791-F801.

14 Deb DK, Sun T, Wong KE, Zhang Z, Ning G, Zhang Y, Kong J, Shi H, Chang A, Li YC: Combined vitamin D analog and AT1 receptor antagonist synergistically block the development of kidney disease in a model of type 2 diabetes. Kidney Int 2010;77:1000-1009.

15 Ohara I, Tanimoto M, Gohda T, Yamazaki T, Hagiwara S, Murakoshi M, Aoki T, Toyoda H, Ishikawa Y, Funabiki K, Horikoshi S, Tomino Y: Effect of combination therapy with angiotensin receptor blocker and 1,25-dihydroxyvitamin $\mathrm{D}(3)$ in type 2 diabetic nephropathy in KK-A(y)/Ta mice. Nephron Exp Nephrol 2011;117:e124-e132.

16 Inukai T, Fujiwara Y, Tayama K, Aso Y, Takemura Y: Alterations in serum levels of 1 alpha,25(OH)2 $\mathrm{D} 3$ and osteocalcin in patients with early diabetic nephropathy. Diabetes Res Clin Pract 1997;38:53-59.

17 de Boer IH, Ioannou GN, Kestenbaum B, Brunzell JD, Weiss NS: 25-Hydroxyvitamin D levels and albuminuria in the Third National Health and Nutrition Examination Survey (NHANES III). Am J Kidney Dis 2007;50:69-77.

18 Diaz VA, Mainous AG,3rd, Carek PJ, Wessell $\mathrm{AM}$, Everett CJ: The association of vitamin D deficiency and insufficiency with diabetic nephropathy: implications for health disparities. J Am Board Fam Med 2009;22:521-527.

19 Damasiewicz MJ, Magliano DJ, Daly RM, Gagnon C, Lu ZX, Ebeling PR, Chadban SJ, Atkins RC, Kerr PG, Shaw JE, Polkinghorne KR: 25-Hydroxyvitamin D levels and chronic kid- ney disease in the AusDiab (Australian Diabetes, Obesity and Lifestyle) study. BMC Nephrol 2012;13:55.

20 Ellam T, Fotheringham J, Wilkie ME, Francis SE, Chico TJ: Bone mineral metabolism parameters and urinary albumin excretion in a representative US population sample. PLoS One 2014;9:e88388.

21 Huang Y, Yu H, Lu J, Guo K, Zhang L, Bao Y, Chen $\mathrm{H}$, Jia W: Oral supplementation with cholecalciferol $800 \mathrm{IU}$ ameliorates albuminuria in Chinese type 2 diabetic patients with nephropathy. PLoS One 2012;7:e50510.

22 Damasiewicz MJ, Magliano DJ, Daly RM, Gagnon C, Lu ZX, Sikaris KA, Ebeling PR, Chadban SJ, Atkins RC, Kerr PG, Shaw JE, Polkinghorne KR: Serum 25-hydroxyvitamin D deficiency and the 5-year incidence of CKD. Am J Kidney Dis 2013;62:58-66.

23 Storm TL, Sorensen OH, Lund B, Lund B, Christiansen JS, Andersen AR, Lumholtz IB, Parving HH: Vitamin D metabolism in insulin-dependent diabetes mellitus. Metab Bone Dis Relat Res 1983;5:107-110.

24 de Boer IH, Sachs MC, Cleary PA, Hoofnagle AN, Lachin JM, Molitch ME, Steffes MW, Sun W, Zinman B, Brunzell JD; Diabetes Control and Complication Trial/Epidemiology of Diabetes Interventions and Complications Study Research Group: Circulating vitamin D metabolites and kidney disease in type 1 diabetes. J Clin Endocrinol Metab 2012;97:47804788.

25 Skaaby T, Husemoen LL, Pisinger C, Jorgensen T, Thuesen BH, Rasmussen K, Fenger $\mathrm{M}$, Rossing $\mathrm{P}$, Linneberg A: Vitamin D status and 5-year changes in urine albumin creatinine ratio and parathyroid hormone in a general population. Endocrine 2013;44:473-480.

26 Kim HW, Park H, Cho KH, Han K, Ko BJ: Parathyroid hormone, vitamin D levels and urine albumin excretion in older persons: the 2011 Korea National Health and Nutrition Examination Survey (KNHANES). Clin Endocrinol (Oxf) 2014;80:34-40.

27 Joergensen C, Hovind P, Schmedes A, Parving $\mathrm{HH}$, Rossing $\mathrm{P}$ : Vitamin D levels, microvascular complications, and mortality in type 1 diabetes. Diabetes Care 2011;34:1081-1085.

28 Jorgensen HS, Winther S, Povlsen JV, Ivarsen $P$ : Effect of vitamin-D analogue on albuminuria in patients with non-dialysed chronic kidney disease stage 4-5: a retrospective single center study. BMC Nephrol 2012;13:102.

29 de Borst MH, Hajhosseiny R, Tamez $\mathrm{H}$, Wenger J, Thadhani R, Goldsmith DJ: Active vitamin $\mathrm{D}$ treatment for reduction of residual proteinuria: a systematic review. J Am Soc Nephrol 2013;24:1863-1871.

30 Larsen T, Mose FH, Bech JN, Pedersen EB: Effect of paricalcitol on renin and albuminuria in non-diabetic stage III-IV chronic kidney disease: a randomized placebo-controlled trial. BMC Nephrol 2013;14:163.

31 Alborzi P, Patel NA, Peterson C, Bills JE, Bekele DM, Bunaye Z, Light RP, Agarwal R: Paricalcitol reduces albuminuria and inflam- mation in chronic kidney disease: a randomized double-blind pilot trial. Hypertension 2008;52:249-255.

32 Hojs N, Bevc S, Balon BP, Hojs R, Ekart R: Paricalcitol reduces proteinuria in non-dialysis chronic kidney disease patients. Ther Apher Dial 2013;17:368-372.

-33 de Zeeuw D, Agarwal R, Amdahl M, Audhya P, Coyne D, Garimella T, Parving HH, Pritchett Y, Remuzzi G, Ritz E, Andress D: Selective vitamin $\mathrm{D}$ receptor activation with paricalcitol for reduction of albuminuria in patients with type 2 diabetes (VITAL study): a randomised controlled trial. Lancet 2010;376: 1543-1551.

34 Maahs DM, Snively BM, Bell RA, Dolan L, Hirsch I, Imperatore G, Linder B, Marcovina SM, Mayer-Davis EJ, Pettitt DJ, Rodriguez BL, Dabelea D: Higher prevalence of elevated albumin excretion in youth with type 2 than type 1 diabetes: the SEARCH for Diabetes in Youth study. Diabetes Care 2007;30:2593-2598.

35 Verrotti A, Basciani F, Carle F, Morgese G, Chiarelli F: Calcium metabolism in adolescents and young adults with type 1 diabetes mellitus without and with persistent microalbuminuria. J Endocrinol Invest 1999;22:198202.

36 Kaur H, Donaghue KC, Chan AK, BenitezAguirre P, Hing S, Lloyd M, Cusumano J, Pryke A, Craig ME: Vitamin D deficiency is associated with retinopathy in children and adolescents with type 1 diabetes. Diabetes Care 2011;34:1400-1402.

37 SEARCH Study Group: SEARCH for Diabetes in Youth: a multicenter study of the prevalence, incidence and classification of diabetes mellitus in youth. Control Clin Trials 2004. 25:458-471.

38 Kershnar AK, Daniels SR, Imperatore G, Palla SL, Petitti DB, Pettitt DJ, Marcovina S, Dolan LM, Hamman RF, Liese AD, Pihoker C, Rodriguez BL: Lipid abnormalities are prevalent in youth with type 1 and type 2 diabetes: the SEARCH for Diabetes in Youth Study. J Pediatr 2006;149:314-319.

39 Yetley EA, Pfeiffer CM, Schleicher RL, et al: NHANES monitoring of serum 25-hydroxyvitamin D: a roundtable summary. J Nutr 2010;140:2030S-2045S.

40 The NS, Crandell JL, Lawrence JM, King IB, Dabelea D, Marcovina SM, D'Agostino RB Jr, Norris JM, Pihoker C, Mayer-Davis EJ: Vitamin $\mathrm{D}$ in youth with Type 1 diabetes: prevalence of insufficiency and association with insulin resistance in the SEARCH Nutrition Ancillary Study. Diabet Med 2013;30:13241332.

41 Chavers BM, Simonson J, Michael AF: A solid phase fluorescent immunoassay for the measurement of human urinary albumin. Kidney Int 1984;25:576-578.

42 Center for Disease Control and Prevention, National Center for Health Statistics: National Health and Nutrition Examination Survey III (1988-1994): Laboratory File Documentation, 2006, 2011. 
-43 Weng FL, Shults J, Herskovitz RM, Zemel BS, Leonard MB: Vitamin D insufficiency in steroid-sensitive nephrotic syndrome in remission. Pediatr Nephrol 2005;20:56-63.

-44 Grymonprez A, Proesmans W, Van Dyck M, Jans I, Goos G, Bouillon R: Vitamin D metabolites in childhood nephrotic syndrome. Pediatr Nephrol 1995;9:278-281.

-45 Freundlich M, Bourgoignie JJ, Zilleruelo G, Jacob AI, Canterbury JM, Strauss J: Bone modulating factors in nephrotic children with normal glomerular filtration rate. Pediatrics 1985;76:280-285.

-46 Freundlich M, Bourgoignie JJ, Zilleruelo G, Abitbol C, Canterbury JM, Strauss J: Calcium and vitamin $\mathrm{D}$ metabolism in children with nephrotic syndrome. J Pediatr 1986;108:383387.

47 Mittal SK, Dash SC, Tiwari SC, Agarwal SK, Saxena S, Fishbane S: Bone histology in patients with nephrotic syndrome and normal renal function. Kidney Int 1999;55:19121919.

-48 Ogden CL, Kuczmarski RJ, Flegal KM, Mei Z, Guo S, Wei R, Grummer-Strawn LM, Curtin LR, Roche AF, Johnson CL: Centers for Disease Control and Prevention 2000 growth charts for the United States: improvements to the 1977 National Center for Health Statistics version. Pediatrics 2002;109:45-60.

49 Center for Disease Control and Prevention, National Center for Health Statistics: National Health and Nutrition Examination Survey, 2005-2006. Data Documentation, Codebook, and Frequencies, 2012, 2014.
50 The National Heart, Lung, and Blood Institute Growth and Health Study Research Group: Obesity and cardiovascular disease risk factors in black and white girls: the NHLBI Growth and Health Study. Am J Public Health 1992;82:1613-1620.

-51 Bonifacio E, Yu L, Williams AK, Eisenbarth GS, Bingley PJ, Marcovina SM, Adler K, Ziegler AG, Mueller PW, Schatz DA, Krischer JP, Steffes MW, Akolkar B: Harmonization of glutamic acid decarboxylase and islet antigen-2 autoantibody assays for national institute of diabetes and digestive and kidney diseases consortia. J Clin Endocrinol Metab 2010;95:3360-3367.

52 Dawson-Hughes B, Heaney RP, Holick MF, Lips P, Meunier PJ, Vieth R: Estimates of optimal vitamin D status. Osteoporos Int 2005; 16:713-716.

53 Pozzilli P, Manfrini S, Crino A, Picardi A, Leomanni C, Cherubini V, Valente L, Khazrai M, Visalli N; IMDIAB group: Low levels of 25-hydroxyvitamin D3 and 1,25-dihydroxyvitamin D3 in patients with newly diagnosed type 1 diabetes. Horm Metab Res 2005; 37:680-683.

54 Littorin B, Blom P, Scholin A, Arnqvist HJ, Blohme G, Bolinder J, Ekbom-Schnell A, Eriksson JW, Gudbjornsdottir S, Nystrom L, Ostman J, Sundkvist G: Lower levels of plasma 25-hydroxyvitamin D among young adults at diagnosis of autoimmune type 1 diabetes compared with control subjects: results from the nationwide Diabetes Incidence Study in Sweden (DISS). Diabetologia 2006; 49:2847-2852.
55 Svoren BM, Volkening LK, Wood JR, Laffel LM: Significant vitamin D deficiency in youth with type 1 diabetes mellitus. J Pediatr 2009; 154:132-134.

56 Skinner AM, Addison GM, Price DA: Changes in the urinary excretion of creatinine, albumin and $\mathrm{N}$-acetyl-beta-D-glucosaminidase with increasing age and maturity in healthy schoolchildren. Eur J Pediatr 1996;155:596602

57 Trachtenberg F, Barregård L: The effect of age, sex, and race on urinary markers of kidney damage in children. Am J Kidney Dis 2007;50:938-945.

58 Hanevold CD, Pollock JS, Harshfield GA: Racial differences in microalbumin excretion in healthy adolescents. Hypertension 2008;51: 334-338.

59 Dodge WF, West EF, Smith EH, Bruce H 3rd: Proteinuria and hematuria in schoolchildren: epidemiology and early natural history. J Pediatr 1976;88:327-347.

60 Vehaskari VM, Rapola J: Isolated proteinuria: analysis of a school-age population. J Pediatr 1982;101:661-668.

61 Rudberg S, Dahlquist G: Determinants of progression of microalbuminuria in adolescents with IDDM. Diabetes Care 1996;19: 369-371.

62 Salardi S, Balsamo C, Zucchini S, Maltoni G, Scipione M, Rollo A, Gualandi S, Cicognani A: High rate of regression from micro-macroalbuminuria to normoalbuminuria in children and adolescents with type 1 diabetes treated or not with enalapril: the influence of HDL cholesterol. Diabetes Care 2011;34:424-429. 